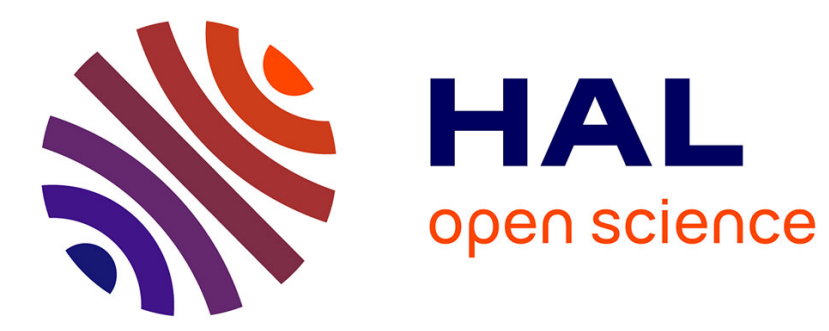

\title{
Romanisation et développement dans les campagnes des Gaules
}

Frédéric Trément

\section{To cite this version:}

Frédéric Trément. Romanisation et développement dans les campagnes des Gaules. Comment les Gaules devinrent romaines, Pierre Ouzoulias, Laurence Tranoy Sep 2007, Paris, France. pp.159-176. halshs-01839301

\section{HAL Id: halshs-01839301 https://shs.hal.science/halshs-01839301}

Submitted on 5 Sep 2018

HAL is a multi-disciplinary open access archive for the deposit and dissemination of scientific research documents, whether they are published or not. The documents may come from teaching and research institutions in France or abroad, or from public or private research centers.
L'archive ouverte pluridisciplinaire HAL, est destinée au dépôt et à la diffusion de documents scientifiques de niveau recherche, publiés ou non, émanant des établissements d'enseignement et de recherche français ou étrangers, des laboratoires publics ou privés. 


\title{
Romanisation et développement dans les campagnes des Gaules
}

Frédéric Trément*

\begin{abstract}
$\longrightarrow$
'est un lieu commun de rappeler que la société gauloise puis gallo-romaine est essentiellement rurale, et que les campagnes y constituent la base de l'économie, comme dans toutes les sociétés traditionnelles jusqu'à la révolution industrielle. Pourtant, la représentation que l'on se fait aujourd'hui des campagnes des Gaules a profondément évolué depuis le XIX siècle, époque à laquelle on les imaginait quasi désertes, occupées seulement par de grandes villae éparses contrôlant d'immenses domaines. Les prospections aériennes conduites par R. Agache dans les années 1970 ont bouleversé cette représentation, remplissant les campagnes d'un nombre considérable d'établissements ruraux, gallo-romains mais également protohistoriques [Agache et Bréart, 1975 ; Agache, 1978]. Dans les années 1980, l'essor des prospections au sol, puis, dans la décennie suivante, celui de l'archéologie préventive et des études paléoenvironnementales ont considérablement affiné notre connaissance des dynamiques et des formes de l'occupation du sol, en particulier pour la Protohistoire. Il en est résulté qu'aujourd'hui les campagnes gauloises de l'âge du Fer
\end{abstract}

* Université Blaise-Pascal - Clermont-Ferrand, Centre d'histoire « Espaces et Cultures ». 
sont aussi bien (ou en tout cas pas plus mal) connues que celles de l'époque romaine.

Cette évolution des connaissances est à l'origine d'un renouvellement profond de la question de la romanisation des campagnes, qui s'inscrit dans un mouvement historique plus large qui porte davantage d'attention aux sociétés indigènes et provinciales intégrées dans l'Empire romain [Wood et Queiroga, 1992; Metzler et al., 1995 ; Webster, 2001 ; Le Roux, 2004 ; Paunier, 2006]. Dès 1993, le $\mathrm{II}^{\mathrm{e}}$ colloque de l'Association d'étude du monde rural gallo-romain (AGER) posait la question de «la romanisation des campagnes de la Gaule » en mettant l'accent sur la continuité « de la ferme indigène à la villa romaine» [Bayard et Collart, 1996]. Les organisateurs du colloque insistaient, dans l'introduction de la publication, sur les nouvelles perspectives théoriques, la complexité des processus, la nécessité d'approches régionales et locales, et l'enjeu que représentent les études paléoenvironnementales. Ils concluaient à la nécessité de rejeter la coupure chronologique marquée par la conquête romaine, prônant l'analyse des dynamiques de l'espace rural dans la longue durée.

Le terme de «romanisation» est, depuis, utilisé avec une précaution accrue. Certains auteurs lui préfèrent, à propos des campagnes de la Gaule, des substitutifs tels qu' «intégration », bien sûr, mais aussi " acculturation » [Ferdière, 2005], ou encore "gallo-romanisation » [Pion, 1996, p. 97]. À la suite de Ph. Leveau [2005a], qui suggère de "déromaniser» l'histoire de l'Afrique romaine, P. Ouzoulias [2007] a milité récemment pour une «déromanisation» des campagnes galloromaines ${ }^{1}$. Je propose d'examiner la question de la romanisation des campagnes dans la longue durée en introduisant la notion de développement. Je limiterai mon propos aux aspects socioéconomiques du développement.

1 Cette formule avait déjà été utilisée par Y. Thébert [1978] à propos de l'Afrique, et par C. Haselgrove et C. Scull [1992] pour la Gaule. 


\section{Des campagnes florissantes et en pleine expansion à l'âge du Fer}

Comme le rappellent les auteurs d'une récente Histoire de l'agriculture en Gaule, ce territoire entre dans l'orbite politique romaine doté d'une agriculture florissante et en pleine expansion [Ferdière et al., 2006]. Dès le premier âge du Fer, la maîtrise de la métallurgie du fer constitue un progrès essentiel, qui conditionne la mise au point d'un outillage agricole et artisanal beaucoup plus résistant et performant que l'outillage de bronze. Le second âge du Fer marque une étape importante de l'histoire de l'agriculture gauloise, qui se traduit par une structuration croissante des paysages agraires [Malrain et al., 2002].

\section{La structuration croissante des paysages agraires}

La croissance démographique est à l'origine d'un développement spectaculaire de l'occupation du sol dans de nombreux secteurs de la Gaule intérieure et méridionale. Elle a pour conséquence la mise en place d'un réseau dense d'exploitations agricoles, dont la cartographie montre la propagation des terres cultivées dès les $\mathrm{V}^{\mathrm{e}}$ et $\mathrm{IV}^{\mathrm{e}} \mathrm{s}$. av. J.-C. $\mathrm{Au} \mathrm{III{ } ^ { e }}$ s., ces exploitations agricoles se généralisent à l'ensemble du paysage. Les données palynologiques confirment l'impact majeur des sociétés de l'âge du Fer sur l'environnement végétal, y compris dans les milieux de montagne (Massif central, Alpes, Jura, Pyrénées).

L'habitat rural est constitué très majoritairement de fermes isolées entourées d'une palissade ou d'un fossé. Olivier Buchsenschutz [1996 ; id., 2004] met ces établissements en rapport avec l'émergence, dans la société gauloise, d'une classe de propriétaires solidement assis sur ces unités de production agricole dispersées dans les campagnes. Il s'agirait des aedificia mentionnés par César dans sa Guerre des Gaules.

Les recherches récentes montrent la diversité jusqu'alors insoupçonnée de ces installations et le caractère trop réducteur de l'expression «ferme indigène », forgée à partir des caractéristiques formelles des sites vus d'avion. Ces établissements liés au mouvement de conquête agraire qui caractérise la fin du second âge du Fer présentent en effet une diversité architecturale plus grande qu'on ne l'imaginait, ainsi que des fonctions variées (habitat, agriculture, mais aussi artisanat) [Marion et Blancquaert, 2000]. Il semble toutefois qu'à La Tène finale on observe dans certaines régions une tendance à la 
standardisation des bâtiments, qui, dans le quart nord-ouest de la France (Bretagne, Normandie, Pays-de-la-Loire et Centre), deviennent majoritairement rectilinéaires à partir du $\mathrm{e}^{\mathrm{er}}$ S. av. J.-C. [Courbot, 2000].

Parallèlement, dans les vallées de la France septentrionale au moins, on constate, à la fin de La Tène, une structuration de plus en plus forte de l'espace environnant l'habitat. L'enclos d'habitat est de plus en plus fortement intégré dans le paysage. Les fouilles et les analyses polliniques conduites sur le tracé de l'autoroute A29, en pays de Caux (Seine-Maritime), illustrent bien cette évolution générale [Rougier, 2000]. À La Tène finale, les établissements s'insèrent dans des parcellaires vastes et complexes qui en prolongent les orientations. La ferme indigène connaît alors une standardisation assez poussée (passage d'enclos ovalaires à des formes plus régulières à La Tène C2/D1). Cette évolution s'accompagne d'un déboisement important et généralisé.

\section{De profondes mutations agricoles à la fin de l'âge du Fer}

Le second âge du Fer correspond également à de profondes mutations agricoles. La maîtrise parfaite de la métallurgie du fer permet de fabriquer des outils très variés et efficaces, qui facilitent les défrichements et améliorent grandement le travail de la terre. La variété des types de socs d'araire est adaptée aux différents types de terrains et de travaux [Marbach, 2004a; id., 2004b]. Au III ${ }^{e}$ s. av. J.-C. apparaît la faux, en lien avec le développement de la production de foin pour l'élevage [Ferdière, 1997 ; Bouby et Ruas, 2005]. Significativement, la longueur de sa lame augmente au siècle suivant. L'une des innovations les plus importantes réside toutefois dans la diffusion de la meule rotative à partir du IV s. av. J.-C. [Amouretti et Comet, 2002]. Empruntée au monde méditerranéen, elle permet de décupler le rendement de la mouture et constitue une vraie révolution technique. Là encore, significativement, on observe un accroissement du diamètre des meules à La Tène finale. Celles-ci sont désormais produites dans des ateliers spécialisés et présentent une relative standardisation.

Les techniques culturales connaissent également d'importants progrès, qui sont à l'origine d'une amélioration des rendements [Ferdière, 1988 ; id., 1991] : généralisation de l'amendement par fumure liée au développement de l'élevage, pratique du chaulage chez les Éduens et les Pictons, du marnage chez les Ubiens selon Pline 
(Histoire naturelle, XVII, 6, $42 ; 7,43 ; 8,47$ ). Le système de culture privilégie l'alternance jachère-céréales d'hiver. Prédominante du viII ${ }^{e}$ au IV ${ }^{e}$ s. av. J.-C. dans le nord de la Gaule, la méture, qui consiste à mélanger plusieurs variétés de plantes dans un même champ pour limiter les effets des aléas météorologiques, régresse au profit des monocultures [Matterne, 2001].

Plus largement, les apports récents de la carpologie mettent en évidence une nette tendance à la spécialisation des cultures. Ils montrent, pour le nord et le centre de la France, comment l'agriculture extrêmement diversifiée du premier âge du Fer se spécialise et s'uniformise à La Tène finale, passant d'une production de subsistance, à l'échelle d'une communauté, à une production pour l'échange basée sur la monospécificité et la spécialisation régionale [Ferdière et al., 2006]. Dans le Midi, en revanche, prédomine une polyculture de complantation basée sur les céréales, les légumineuses et les vergers [Marinval, 2004]. La viticulture, qui apparaît en contexte indigène dès la fin du ve s. av. J.-C. en Languedoc (Lattes), se développe surtout à partir du III s. [Brun et Laubenheimer, 2001]. D'autres arbres fruitiers font leur apparition (olivier, figuier, grenadier). L'oléiculture, moins développée que la viticulture, se répand à partir du $\mathrm{III}^{\mathrm{e}} \mathrm{s}$. en lien avec Marseille [Brun, 2003 ; id., 2005].

Des tendances similaires s'observent dans les progrès de l'élevage : amélioration de l'alimentation grâce au développement du fourrage, diversification des espèces, régionalisation [Arbogast et al., 2006]. La faible taille et la gracilité des animaux limitent toutefois leur rendement (force de traction, bât, lait, laine, viande) et posent problème pour l'exploitation des terres lourdes. Or, dès le milieu du III ${ }^{e} s$. av. J.-C., on observe une amélioration de l'élevage dans le Midi (Lattes), avec une augmentation de la stature moyenne des bêtes. L'introduction précoce de bœufs et de chevaux de grande taille dans le reste de la Gaule à la fin du II $\mathrm{s}$. av. J.-C. constitue un progrès essentiel. Plus puissant, le grand bœuf fournit aussi plus de viande. L'évolution rapide de ce phénomène prouve qu'il s'agit d'un apport extérieur et non du résultat d'une sélection [Forest et Rodet-Belarbi, 2002]. Sa concomitance avec la conquête romaine de la Gaule du Sud suggère une importation d'origine italienne. On observe, parallèlement, une nouvelle organisation, plus spécialisée, de la boucherie et du commerce des viandes en Gaule à la fin de l'âge du Fer [Salin, 2007], 
qui a des répercussions sur l'artisanat qui en découle (travail du cuir, de la fourrure, de la corne et de l'os).

Ces innovations techniques permettent un net accroissement des rendements et de la production durant les trois siècles avant le changement d'ère. L'étude des modalités de stockage confirme la forte hausse de la production, la plus forte hiérarchisation des habitats et la spécialisation accrue d'une partie des fermes indigènes dans la production agricole. Les recherches de F. Gransar [2002] sur le stockage alimentaire dans les établissements ruraux mettent en évidence la diversité et la complémentarité des différents modes de stockage : silos enterrés, greniers surélevés sur poteaux, conteneurs en céramique ou en torchis. Les silos sont utilisés pour stocker de grandes quantités de céréales en vue d'un usage différé et communautaire, tandis que les greniers répondent aux besoins courants. La période du Hallstatt final et de La Tène ancienne se caractérise par un fort dégagement de surplus sur certains sites, par l'apparition d'espaces spécialisés au sein des habitats, par une bipartition entre sites producteurs et sites centralisateurs de récoltes. À La Tène finale, la raréfaction des moyens de stockage sur les établissements ruraux s'explique par la concentration des récoltes sur les oppida.

Les progrès de l'exploitation du sel constituent un autre indice, indirect, de l'accroissement de la production agricole. L'augmentation de la production de sel vise en effet à répondre aux besoins de conservation accrus. La technique du bouillage en augets de terre cuite, appelés briquetages, est aujourd'hui mieux connue. Les travaux de l'autoroute A16 ont mis en évidence une chaîne opératoire très élaborée [Prilaux, 2000]. La typo-chronologie des fourneaux et du mobilier montre comment le procédé ancien hérité de l'âge du Bronze évolue au second âge du Fer, avec l'apparition de grands fourneaux à grille qui autorisent un accroissement très important de la production à la fin de La Tène. Dès le III ${ }^{e}$ s. av. J.-C., les sauniers transforment radicalement leurs méthodes de travail en rationalisant les procédés et en intégrant les ateliers à l'habitat. Ces évolutions sont liées à l'augmentation et à la diversification de la demande : le sel n'est pas seulement un agent conservateur, il est également utilisé dans l'artisanat (orfèvrerie, traitement des peaux, teinture, verrerie, métallurgie, fromagerie, pharmacie), sans parler de sa valeur d'échange intrinsèque [Weller, 2000]. 
L'un des traits les plus marquants de l'évolution économique réside dans le développement considérable des échanges, qui s'amplifient avec la mainmise de Rome sur la Transalpine à la fin du II $\mathrm{s}$. av. J.-C. On sait que des marchands romains sont installés en Gaule intérieure avant la conquête césarienne [Goudineau, 1998a]. César, Diodore et Strabon, qui puisent à la même source, Poséidonios, soulignent les avantages que procure à la Gaule le réseau développé de voies fluviales et terrestres. Le monnayage celtique apparaît dès la fin du III $s$. Mais la circulation monétaire reste encore limitée et n'augmente qu'à partir du début du $\mathrm{I}^{\mathrm{er}} \mathrm{S}$. av. J.-C., avec la diffusion des petits bronzes coulés [Guichard et al., 1993]. Ce phénomène est toutefois concentré dans les grosses agglomérations de commerçants et d'artisans. L'importation massive de vin campanien et étrusque pose le problème des contreparties, plus difficiles à mettre en évidence par l'archéologie. Les textes évoquent les esclaves, les produits miniers et agricoles [Tchernia, 1986]. Les protohistoriens ont souligné depuis longtemps le rôle central joué par les oppida dans ces échanges. Les oppida sont à la fois des lieux de centralisation des surplus de la production agricole et de redistribution des produits importés [Audouze et Buchsenschutz, 1989].

\section{Après la conquête romaine : rupture ou continuité?}

\section{Quelle romanisation après la Conquête?}

L'évolution des campagnes dans les décennies qui suivent la conquête par César est malheureusement documentée de manière très disparate. Il en va différemment à partir du changement d'ère. Le $\mathrm{I}^{\text {er }} \mathrm{s}$. correspond partout à un mouvement général de densification de l'habitat rural, qui se poursuit et culmine au siècle suivant. A. Ferdière [2005] estime la population rurale de la Gaule à 11 millions d'habitants, ce qui implique une densité moyenne élevée. Les cartes archéologiques montrent toutefois d'importantes variations d'une région à l'autre, et à l'intérieur d'une même région. L'hétérogénéité des données, celle des méthodes et des définitions limitent cependant les possibilités de comparaison. On retiendra que de nombreuses zones géographiques sont très densément occupées et mises en valeur, en particulier dans les grandes plaines (fig. 1). 


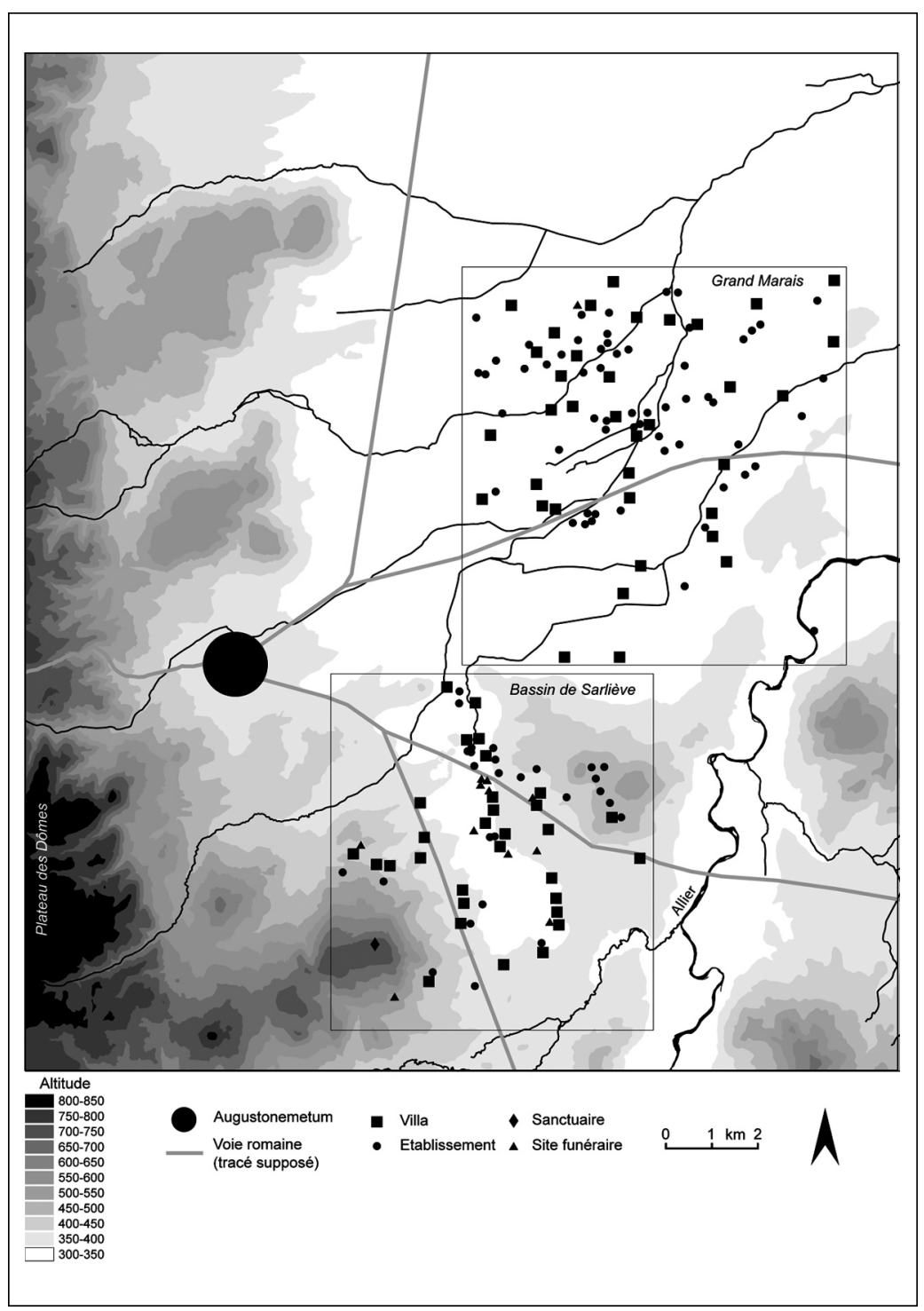

Fig. 1. Le bassin de Clermont-Ferrand au Haut-Empire. Les prospections systématiques effectuées dans le Grand marais et le bassin de Sarliève révèlent une densité très élevée d'établissements agricoles (villae et fermes) régulièrement répartis dans l'espace (un site tous les $250 \mathrm{~m}$ ) (F. Trément). 
D’une manière générale, aucune zone géographique n'est vide d'occupation. Les recherches paléoenvironnementales récentes invitent néanmoins à relativiser l'importance de l'exploitation de la haute montagne, par rapport à l'époque médiévale, qui marque une intensification sans précédent de la mise en valeur agropastorale de ces milieux [Galop, 1998 ; Miras, 2004 ; Segard, 2009]. Il semble que l'époque romaine se caractérise surtout, plus que par une extension des zones cultivées, par une intensification de l'exploitation des terroirs déjà déboisés. L'une des questions qui se posent actuellement est de savoir si de nouveaux espaces sont mis en valeur durant l'époque romaine. Dans les milieux «marginaux » de marais, de forêts et de moyenne montagne, les méthodes de prospection archéologique se heurtent à la surreprésentation des sites d'époque romaine, plus facilement identifiables que les établissements protohistoriques. Cette question $\mathrm{du}$ «front pionnier » a fait l'objet de débats animés ces dernières années [Leveau, 2005b]. Comme on l'a vu, les études paléoenvironnementales montrent que le déboisement est déjà très poussé un peu partout dans les plaines dès l'âge du Fer [Frenzel, 1994 ; Chabal, 1997 ; Vernet, 1997 ; Antoine et Marguerie, 2007]. Le problème de l'exploitation des massifs forestiers subsistants reste entier, tant pour l'âge du Fer que pour l'époque romaine [Nenninger, 2001]. Mais il est certain que les besoins en bois se sont accrus après la Conquête : bois d'œuvre pour la construction (y compris navale), combustible pour le chauffage et l'artisanat, matière première pour l'artisanat (que l'on pense aux besoins de la tonnellerie et de la charronnerie), charbon de bois pour la réduction du fer, poix pour le calfat, le poissage des amphores et des dolia [Bernard, 1998 ; Trintignac, 2003].

Dans le domaine de l'outillage et de l'équipement agricole, les innovations postérieures à la Conquête apparaissent pourtant plus limitées qu'à La Tène [Ferdière et al., 2006]. Des améliorations sont apportées à l'araire, qui est doté d'oreilles faisant office de versoir, peut-être parfois d'un coutre en fer. L'utilisation de la herse constitue une nouveauté. Celle du vallus, qui a été présenté comme une révolution technique, est en fait mal datée et paraît limitée aux grandes plaines du Nord-Est (Trévires et Rèmes d'après l'iconographie). On signalera encore l'apparition des bousandales et du fer à cheval, ou encore le net développement de l'usage des mules et mulets pour le transport, ce qui implique des élevages d'ânes. L'une des innovations 
gauloises les plus importantes est le tonneau, dont la diffusion devient effective à partir de la seconde moitié du $\mathrm{I}^{\mathrm{er}} \mathrm{S}$. [Marlière, 2001 ; id., 2002].

C'est dans le domaine de la transformation des produits agricoles qu'ont lieu certainement les progrès les plus importants, avec l'introduction des meules asinaires (dites pompéiennes) et celle, surtout, de moulins hydrauliques, dont la diffusion apparaît beaucoup plus développée qu'on ne le pensait : en effet, presque toutes les villae importantes devaient en disposer [Brun et Fiches, 2007]. Le plus ancien moulin à eau reconnu à ce jour en Gaule, près de Bourges, date du début du $\mathrm{er}^{\text {er }} \mathrm{s}$. [Champagne et al., 1997]. La meunerie de Barbegal, près d'Arles, reste toutefois, par ses dimensions imposantes, un unicum dans le monde romain [Leveau, 2007]. Parallèlement, le développement important de la viticulture et, dans une mesure bien moindre, de l'oléiculture, s'accompagne d'une banalisation des systèmes de pressage maçonnés.

Dans le domaine des techniques culturales, on note la disparition de la méture et la diffusion de la rotation triennale des cultures. Mais on n'a, à ce jour, aucune preuve de la pratique de l'assolement biennal ou triennal. L'irrigation, considérée comme une innovation de l'époque romaine, paraît avoir été limitée aux jardins et potagers de la Gaule du Sud ${ }^{2}$. Quant aux techniques de drainage, il semble qu'elles aient été développées dès le second âge du Fer à une échelle relativement large dans certaines régions de Gaule. Dans le Grand marais de Limagne, en territoire arverne, les fouilles de l'A710 révèlent que le drainage artificiel de la plaine débute dès la seconde moitié du III ${ }^{e} \mathrm{~S}$. av. J.-C. [Guichard, 2000 ; Trément et al., 2004].

Durant le Haut-Empire, le stockage des moissons est effectué exclusivement dans des greniers aériens et des granges, et non plus dans des silos enterrés. Cette évolution est amorcée dès La Tène finale, mais l'époque romaine voit le développement de vastes greniers à structure massive maçonnée, dont la capacité de stockage est sans commune mesure, tout comme celle des horrea urbains.

En définitive, on peut considérer que la période qui suit la conquête romaine n'est marquée dans les campagnes de Gaule par

2 L'irrigation est attestée au Haut-Empire par une canalisation maçonnée dans le Grand marais de Limagne [Guichard 2000], mais rien n'indique qu'elle y ait été pratiquée sur une grande échelle. 
aucune révolution dans le domaine technique, à l'exception peut-être du moulin hydraulique. C'est ailleurs qu'il faut rechercher les évolutions qui ont le plus d'incidences sur le développement des campagnes, en particulier dans la structuration des territoires, qui s'opère avec la mise en place d'un réseau de chefs-lieux, de villes, d'agglomérations, de routes, d'ouvrages d'art divers, de canaux et de ports. Ces infrastructures donnent souvent naissance à de nouvelles places de commerce, tout comme les villes et les agglomérations dites "secondaires », qui jouent un rôle essentiel dans les relations villescampagnes. De vastes travaux d'aménagement du territoire affectent directement les campagnes, notamment dans les terres coloniales, avec la mise en place de centuriations en Narbonnaise. Mais, d'une manière générale, le développement des campagnes gallo-romaines est avant tout la conséquence de l'intégration du territoire gaulois dans $l^{\prime}$ «économie-monde » romaine, mouvement qui est accéléré par l'intensification des échanges, la monétarisation de l'économie et la mise en place de ces nouvelles infrastructures.

La conséquence principale de cette évolution générale est l'augmentation de la production et de la productivité agricoles. Celles-ci passent par une intensification de la mise en valeur des terres et par une spécialisation accrue des cultures [Ferdière et al., 2006]. La monoculture semble demeurer toutefois une tendance, rarement exclusive, qui pose la question de la spécialisation plus ou moins poussée des exploitations agricoles. Le développement d'une viticulture spéculative de masse en Narbonnaise à partir de la seconde moitié du $\mathrm{I}^{\text {er }} \mathrm{s}$. et au siècle suivant n'a pas d'autre équivalent en Gaule, sauf peut-être dans le Nord, où la céréaliculture domine largement le paysage.

Il convient de souligner l'intérêt majeur des approches archéobotaniques et archéozoologiques pour ces questions [Lepetz et al., 2002]. Il est également nécessaire de souligner les limites auxquelles l'archéologie est confrontée pour aborder le problème de la commercialisation des productions agricoles : problème de la «traçabilité » des exportations céréalières, problème du tonneau comme marqueur du commerce des vins gallo-romains et, à l'inverse, surreprésentation des céramiques et des amphores. Dans le même ordre d'idées, la disparition de la technique des briquetages protohistoriques, vraisemblablement au profit des marais salants, rend impossible l'évaluation de la production de sel nécessaire pour la conservation et le transport de nombreuses productions agricoles. 
Il faudrait aborder aussi la question de la place de l'artisanat dans les campagnes, certainement sous-estimée, et pourtant partie intégrante du domaine rural : production de matières premières, productions manufacturées, transformation des produits agricoles [Polfer, 1999 ; Domergue et Leroy, 2000 ; Lepetz et Matterne, 2003]. Ces activités ont des incidences directes sur le développement de l'économie rurale et, plus largement, des campagnes.

La villa, marqueur du développement des campagnes gallo-romaines?

La question de la villa se trouve au cœur du débat sur la romanisation des campagnes de la Gaule. C'est d'elle qu'a surgi récemment l'idée de les «déromaniser». Posons le problème de la manière suivante : dans quelle mesure la villa est-elle un marqueur du développement des campagnes gallo-romaines?

La tradition historiographique française accorde depuis le XIX $\mathrm{s}$. à la villa un rôle central dans l'organisation et la mise en valeur des campagnes gallo-romaines [Leveau et al., 1999b ; Ouzoulias, 2006]. Cette position s'appuie sur la présence massive de la villa sur l'ensemble du territoire. La villa est perçue comme l'instrument privilégié de la mise en valeur des campagnes par les élites provinciales, dans le cadre du domaine. À cette économie domaniale correspondrait un mode d'exploitation de type romain, que refléterait la bipartition pars urbana/pars rustica, et qui aurait pour finalité la rationalisation de la production, destinée à la commercialisation dans le cadre de l'économie d'échanges [Leveau et al., 1993]. Ce système de production accorde une place centrale aux élites foncières, qui sont en même temps les notables municipaux qui monopolisent le pouvoir dans la cité. Ainsi la villa serait-elle la marque par excellence de la romanisation des campagnes de la Gaule.

Depuis quelques années, la place et le rôle de la villa dans les campagnes gallo-romaines ont été revus à la baisse par bon nombre de chercheurs. L'archéologie aérienne, on l'a vu, n'est pas étrangère à ce changement de perspective, puisqu'elle a révélé, en même temps que des «villae romaines », d'innombrables «fermes indigènes ». Mais ce sont surtout le développement de l'archéologie préventive dans les grandes plaines du nord et de l'ouest de la France, et les progrès qu'il a entraînés dans la connaissance des fermes gauloises, qui ont complètement renversé la perspective sur l'origine de la villa. A. Grenier [1934] 
avait remarqué, à la suite de F. CElmann [1928], que bon nombre de villae succèdent à des établissements agricoles laténiens. Mais il voyait dans ce phénomène la succession de deux types d'organisation complètement différents. Le colloque AGER « De la ferme indigène à la villa romaine » a été au contraire l'occasion de mettre en avant «une filiation directe entre la "ferme indigène" et la villa», posant ainsi le problème de la signification socioéconomique de cette dernière [Bayard et Collart, 1996, p. 5]. Pour D. Bayard et J.-L. Collart, les villae de Picardie ne sont qu'en apparence des ensembles architecturaux more romano, car ce sont en réalité majoritairement des créations locales élaborées à partir de modèles indigènes. La villa serait en quelque sorte un «habillage à la romaine » d'une forme d'exploitation préexistante. À l'appui de ce raisonnement, la fouille du site de Verneuil-en-Halatte (Picardie) offrait un exemple remarquable de succession d'une villa à une ferme indigène construite en bois, dont elle épouse le plan [Collart, 1996].

En Picardie, les fermes gallo-romaines précoces feraient ainsi la transition entre la ferme indigène et la villa à cour centrale. Elles prolongeraient, un siècle après la Conquête, un type de structure apparu à la fin de l'âge du Fer. Or, malgré la grande continuité de l'organisation spatiale de certains établissements comme celui de Famechon (sur plus de quatre siècles), les fouilleurs observent, à partir du milieu du $\mathrm{I}^{\text {er }}$ s. apr. J.-C., des transformations considérables dans la nature de leur occupation (plan des bâtiments, techniques de construction). Il y a apparemment rupture entre la ferme galloromaine précoce et la villa classique, même si cette dernière conserve les grandes lignes de l'organisation spatiale antérieure [Collart, 1996]. En outre, à côté de ces fermes gallo-romaines précoces, se développent de grands établissements gallo-romains caractérisés par leur ampleur, leur organisation et l'absence d'occupation antérieure. La question se pose de savoir si cette diversité des formes de l'habitat au début du $\mathrm{I}^{\text {er }} \mathrm{S}$. reflète des formes économiques différentes, ou bien des comportements différenciés des élites foncières face au modèle architectural romain.

Les protohistoriens ont utilisé ces exemples pour relativiser la rupture introduite par la romanisation dans les campagnes gauloises. Ainsi, P. Pion [1996, p. 97] a pu écrire : 
La villa apparaît moins comme le signe d'une irruption soudaine de la romanité et d'un bouleversement brutal des formes de production agricole que comme l'aboutissement d'une différenciation et d'une hiérarchisation de l'habitat et de la société rurale indigènes entamées de longue date et précipitées par l'ampleur que prennent alors le développement urbain et les nouveaux débouchés qui se créent.

L'adoption des formes romaines de l'habitat aurait ainsi permis aux élites foncières de renforcer leur pouvoir sur les campagnes tout en affichant les apparences de la romanité. Ce raisonnement a été largement développé par G. Woolf [1998] dans son ouvrage Becoming Roman. La véritable rupture se situerait au II ${ }^{\mathrm{e}}$ s. av. J.-C., avec le développement conjoint des fermes indigènes et des oppida, qui préfigurent, selon P. Pion, le couple ville-campagne.

Depuis, d'autres arguments sont venus relativiser la place de la villa dans les campagnes gallo-romaines : d'une part, le progrès des connaissances sur les agglomérations secondaires ; d'autre part, la mise en évidence, par l'archéologie préventive, d'une diversité plus grande que l'on ne l'imaginait des formes de l'habitat rural gallo-romain. Symptomatiquement, le dernier colloque AGER avait pour but de faire le point sur les questions de terminologie et de typologie [Leveau et al., 2009]. Il a montré à quel point nos catégories (villae, fermes, agglomérations) sont figées, rendant atypiques de nombreuses exploitations qualifiées timidement de fermes. Ce colloque a également révélé, si besoin en était, la diversité des situations régionales et intrarégionales. Plus que jamais, il apparaît que la réalité de la villa nous échappe, ce qui ne signifie pas, bien sûr, qu'il faille la nier. On ne peut même que souhaiter la fouille exhaustive de ce type d'établissement. Mais il est également nécessaire de ne pas se limiter à des monographies de sites, insuffisantes pour comprendre la dynamique des systèmes de peuplement. Des approches microrégionales sont indispensables pour éclairer les relations entre les différentes formes de l'habitat dans un espace donné et à travers le temps. Et, là encore, l'apport des disciplines paléoenvironnementales est crucial pour accéder à l'objet même qui nous préoccupe, comme il préoccupait les agriculteurs gallo-romains : le sol !

Les recherches conduites en Grande Limagne montrent que, même dans un secteur largement dominé par la villa, ce mode d'exploitation du sol présente une grande diversité à l'échelle microrégionale [Dousteyssier et Trément, 2007] (fig. 2). De toute évidence, la typologie, la 


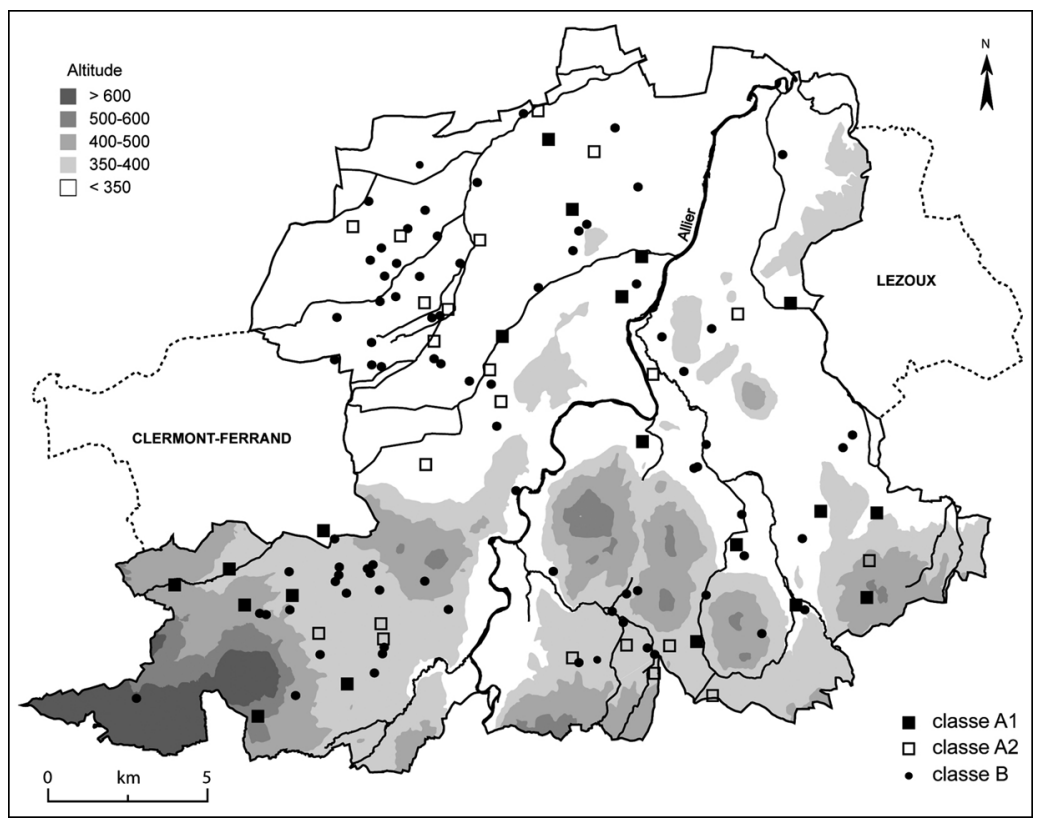

Fig. 2. Typologie et géographie des villae de Grande Limagne au Haut-Empire [d'après Dousteyssier et al., 2004]. Cette carte, fondée sur des prospections systématiques pédestres et aériennes, montre un lien entre typologie et répartition spatiale des villae.

Classe $\mathrm{A} 1$ : Très grosses villae ( 1,8 ha en moyenne) à occupation longue (Haut et Bas-Empire), présentant un niveau élevé de standing (thermes, marbre abondant, mosaïque, enduits peints). Ces établissements espacés de 2 à $3 \mathrm{~km}$ se localisent préférentiellement au sud d'Augustonemetum et au sud de Lezoux. Ils sont absents des zones les plus basses (Grand marais).

Classe A2 : Grosses villae (0,8 ha en moyenne), présentant un niveau de standing moins élevé (présence de thermes, mais marbre plus rare). Ces établissements, qui s'intercalent entre ceux de la classe A1, sont implantés dans tous les types de terroirs.

Classe B : Petites villae (0,3 ha en moyenne), présentant un standing limité (hypocauste, enduits peints). Ces établissements constituent un réseau homogène, particulièrement dense dans les zones basses (Grand marais). 
chronologie et la distribution spatiale des villae tiennent compte des modes d'occupation antérieurs, remontant à La Tène finale, mais aussi de la structuration nouvelle du territoire arverne, marquée par la fondation du chef-lieu de cité Augustonemetum, par le développement du réseau routier et par l'émergence du complexe potier de Lezoux [Dousteyssier et al., 2004]. Mais il est tout aussi évident que le développement de la villa dans cette plaine traduit également une logique de rationalisation et d'intensification de la mise en valeur des riches terres noires [Trément, 2004 ; id., 2007] (fig. 3).

Pour revenir à la question posée plus haut - la villa est-elle un marqueur du développement des campagnes gallo-romaines? -, on répondra par l'affirmative. Mais il est certain que la villa n'est pas le seul acteur du développement de l'espace rural, qui s'effectue dans un contexte plus large.

\section{Conclusion : Pour une analyse des processus de développement régional dans la longue durée}

La « romanisation » apparaît en effet comme un processus de diversification et de complexification dans l'organisation des campagnes de la Gaule, ce que l'on peut considérer comme un développement, au sens où ses modalités sont à la fois socioéconomiques et culturelles. Le concept de développement a l'avantage, sur celui de romanisation, d'une plus grande neutralité idéologique, tout en obligeant le chercheur à expliquer des processus dans leur dimension spatiale autant que temporelle. L'un des enjeux de la problématique du développement est de mettre en évidence en quoi la « romanisation » est facteur non pas d'homogénéisation mais de diversification régionale [Leveau, 2003 ; id., 2007b].

Si l'on examine la « romanisation » des campagnes de la Gaule sous cet angle, dans la longue durée, on peut distinguer trois phases successives. La première, antérieure à la conquête romaine de la Gaule du Sud, se caractérise par des processus de développement endogènes, internes au monde celtique, nourris d'emprunts techniques au monde méditerranéen. La seconde phase, qui correspond grosso modo à la période qui s'écoule entre la conquête de la Gaule du Sud et la période 


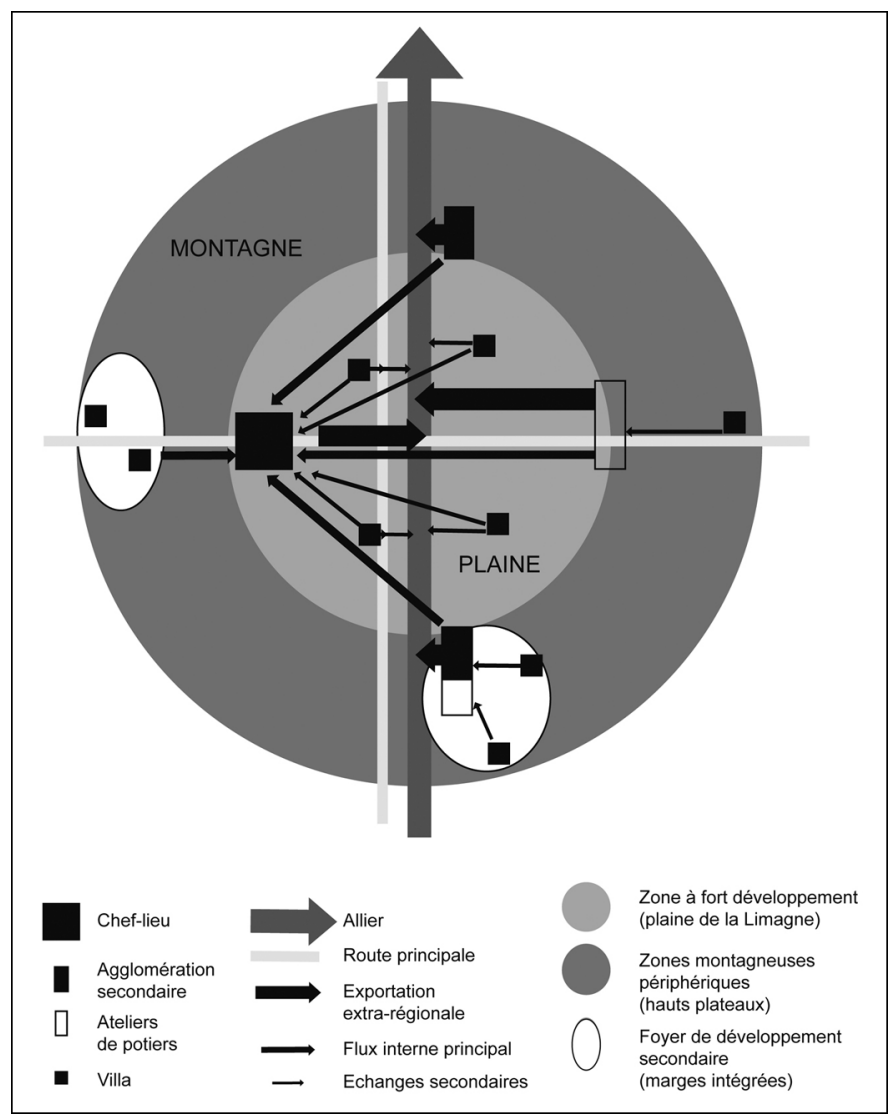

Fig. 3. Essai de modélisation de l'organisation du territoire proche d'Augustonemetum au Haut-Empire. Ce modèle met en relation l'espace géographique, les formes de l'habitat et les flux liés aux surplus de production. Il fait apparaître trois zones de développement inégal à l'échelle régionale : la plaine de la Limagne, caractérisée par une mise en valeur intensive dans le cadre de l'économie domaniale ; les zones montagneuses périphériques (plateau des Dômes, Combrailles, Livradois, Forez), moins densément exploitées, par un tissu plus lâche de villae et d'agglomérations ; à l'intérieur de ces zones périphériques, des foyers de développement (marges intégrées) favorisés par la proximité d'axes de communication importants (voies, rivière Allier). Ce modèle de développement s'appuie sur l'hypothèse d'un système de production excédentaire à l'échelle régionale (céréales, céramiques sigillées) (F. Trément). 
augustéenne (au sens large), se caractérise en Gaule intérieure par une intensification des échanges (de produits mais aussi d'hommes, de connaissances et d'idées), et par des emprunts plus directs et plus massifs au modèle romain ; ces emprunts contribuent à accélérer les processus de développement déjà à l'œuvre dans les campagnes gauloises. Enfin, la troisième phase, marquée par l'intégration politique des Gaules dans l'Empire, est caractérisée par un mode de développement "gallo-romain », dont les spécificités, par rapport aux provinces voisines, gagneraient à être mieux définies.

On a donc affaire à deux modes de développement, l'un gaulois et l'autre romano-méditerranéen, qui exercent l'un sur l'autre des influences croissantes, puis deviennent de plus en plus interdépendants à mesure que la sphère d'influence romaine s'étend autour de la Méditerranée. Dans ce processus, les élites, gauloises puis galloromaines, jouent un rôle moteur. Ce sont elles qui, à l'âge du Fer, contrôlent les échanges et donc l'ouverture aux influences méditerranéennes. Ce sont elles qui, après la Conquête, sont en premier intégrées, romanisées. Or il s'agit avant tout de propriétaires fonciers, qui véhiculent dans les campagnes innovations et nouveaux modèles. De ce point de vue, l'idée d'une inertie du monde rural doit être combattue. Le dynamisme de l'agriculture gauloise au second âge du Fer témoigne au contraire de sa capacité à évoluer rapidement.

Il ne faudrait pas que le débat actuel autour de la villa minimise le rôle de ces élites dans les processus de développement des campagnes gallo-romaines. N'oublions pas que, même si elles contrôlent d'autres sources de richesse (artisanat, commerce, industrie), leur fortune est aussi (et même majoritairement) assise sur l'exploitation de la terre. Sidoine Apollinaire, six siècles après la conquête des Gaules, nous rappelle le poids de cette idéologie aristocratique attachée au domaine terrien. Nul doute, par conséquent, que ces propriétaires gallo-romains n'aient cherché à tirer le meilleur profit de ce modèle d'exploitation présent partout autour de la Méditerranée. La villa s'inscrit dans une stratégie d'intégration et d'adaptation des élites au nouveau contexte économique « mondial ». 